\title{
An Optimal Monte Carlo algorithm for a Class of Multidimensional Integrals
}

\author{
Ivan Dimov \\ Bulgarian Academy of Sciences
}

Venelin Todorov

Bulgarian Academy of Sciences

Institute of Mathematics and Informatics

ul. G. Bonchev 8, 1113 Sofia, Bulgaria

Bulgarian Academy of Sciences

Institute of Information and Communication Technologies

ul. G. Bonchev 25A, 1113 Sofia, Bulgaria

Email: vtodorov@math.bas.bg,venelin@ parallel.bas.bg

Institute of Information and Communication Technologies

ul. G. Bonchev 25A, 1113 Sofia, Bulgaria

Email: ivdimov@bas.bg

Stoyan Poryazov

Bulgarian Academy of Sciences

Institute of Mathematics and Informatics

ul. G. Bonchev 8, 1113 Sofia, Bulgaria

Email: stoyan@math.bas.bg

\author{
Stoyan Apostolov \\ Sofia University \\ Faculty of Mathematics and Informatics \\ 1764 Sofia, Bulgria \\ Email: stoyanrapostolov@gmail.com
}

\author{
Stefka Fidanova \\ Bulgarian Academy of Sciences \\ Institute of Information and Communication Technologies \\ ul. G. Bonchev 25A, 1113 Sofia, Bulgaria \\ Email: stefka@parallel.bas.bg
}

\author{
Yuri Dimitrov \\ Department of Mathematics and Physics \\ University of Forestry \\ Sofia 1756, Bulgaria \\ Bulgarian Academy of Sciences \\ Institute of Mathematics and Informatics \\ Sofia 1113, Bulgaria \\ Email: yuri.dimitrov@ltu.bg
}

\begin{abstract}
An optimal stochastic approach for multidimensional integrals of smooth functions. This is the first time this optimal stochastic approach has been compared with other stochastic approaches for mid and high dimensions. The purpose of the present study is to compare the optimal algorithm with the lattice rules based on the generalized Fibonacci numbers of the corresponding dimension and to discuss the advantages and disadvantages of each method.
\end{abstract}

\section{INTRODUCTION}

The Monte Carlo method has proven to be very useful tool for numerical analysis, particularly when the number of dimension ranging from medium to large. Monte Carlo simulation and quasi-Monte Carlo methods are the prevailing methods used to solve multi-dimensional problems in different areas. Both methods do not suffer from the ,curse of the dimensionality" [2] This is the first time to study optimal

Stoyan Apostolov and Yuri Dmitrov are supported by the Bulgarian National Science Fund under Young Scientists Project KP-06-M32/2 17.12.2019 "Advanced Stochastic and Deterministic Approaches for LargeScale Problems of Computational Mathematics". Venelin Todorov is supported by the National Scientific Program "Information and Communication Technologies for a Single Digital Market in Science, Education and Security (ICT in SES)", contract No DO1-205/23.11.2018, financed by the Ministry of Education and Science in Bulgaria and by the National Scientific Program for post doctoral and young scientists of Ministry of Education and Science 2020-2021. stochastic algorithms for computing multidimensional integrals of smooth functions. The optimal approach has been established by Atanasov and Dimov in [1] but there aren't any comparison with other stochastic approaches up to now.

\section{Description of the Optimal Monte Carlo ALGORITHM}

Most Monte Carlo methods improve achieves better convergence using the idea of dividing the area of integration [2]. In the case where, in addition to the idea of dividing the area $\Omega=U^{s}$ (but without the recursive element), the information on the smoothness of the subintegral function is used, an increase in the order of convergence is achieved. Our first known results in this area with probability density $p(\mathbf{x})=1$, and splitting the area into equal parts in all directions are obtained by Dupach ([4]).

Theorem 1: (Dupach, [4]). Let $g(\mathbf{x})$ and all its first-order partial derivatives $\frac{\partial g}{\partial x_{k}}$ are continuous in $\Omega$ and bounded, i.e. for all $1 \leq k \leq s:\left|\frac{\partial g}{\partial x_{k}}\right| \leq L$, and there exist constants $c_{1}, c_{2}>0$, for which are fulfilled the following conditions

$$
p_{j} \leq \frac{c_{1}}{N}, \quad j=1, \ldots, N, \quad d_{j} \leq \frac{c_{2}}{N^{1 / s}},
$$


where $d_{j}$ is the diameter of the subdomain $\Omega_{j}$, i.e. $d_{j}=$ $\sup \left|\mathbf{x}_{1}-\mathbf{x}_{2}\right|$. Then for the estimation of the variance $\mathbf{x}_{1}, \mathbf{x}_{2} \in \Omega_{j}$

we obtain $\bar{\theta}_{N}^{*}$ in the case of $M=N \quad N_{j}=1, j=1, \ldots, M$ and:

$$
\mathbf{D} \bar{\theta}_{N}^{*} \leq c^{2} L^{2} N^{-1-2 / s}, \quad \text { where } \quad c=s c_{1} c_{2} .
$$

Using Chebyshev's inequality [?], [?], for the error $\bar{\theta}_{N}^{*}-I[g]$ we obtain

$$
P\left\{\left|\bar{\theta}_{N}^{*}-I[g]\right|<\frac{c L}{\varepsilon} N^{-1 / 2-1 / s}\right\} \geq 1-\varepsilon^{2},
$$

where $\varepsilon$ is a small enough positive number.

Given the equation (1) it can be concluded that the probable error $\bar{\theta}_{N}^{*}-I[g]$ decreases in order $N^{-1 / 2-1 / s}$, i.e. faster than $N^{-1 / 2}$. Obviously for large dimensions $s$ the acceleration of the convergence order is relatively insignificant.

The same result for the order of convergence can be achieved in less favorable conditions, namely, only that the corresponding function is continuous. The proof is made by Dimov and Tonev ([3]), and the statement is formulated in the following theorem:

Theorem 2: (Dimov, Tonev; [3]). Let $g$ is continuous in $\Omega \subset \mathbf{R}^{s}$ and there exist constants $c_{1}, c_{2}, c_{3}$, such that $p_{j} \leq$ $c_{1} / N, d_{j} \leq c_{2} N^{-1 / s}$ and $S_{j}\left(\cdot, c_{3}\right) \subset \Omega_{j}, j=1, \ldots, N$ (i.e. $M=N$ and $\left.N_{j}=1\right)$, where $S_{j}\left(\cdot, c_{3}\right)$ is sphere with radius $c_{3}$. Then for the probable error $r_{N}$ is fulfilled [2]:

$$
r_{N} \leq 4 \sqrt{2} \frac{c_{1} c_{2}}{c_{3}} \tau\left(g ; d_{\max }\right)_{L_{q}} N^{-1 / 2-1 / s},
$$

where $d_{\max }=\max _{j} d_{j} \tau(g ; \delta)_{L_{q}}$ is the average modulus of continuity, that is,

$$
\begin{gathered}
\tau(g ; \delta)_{L_{q}}=\|\omega(g, \cdot ; \delta)\|_{L_{q}}=\left(\int_{\Omega}(\omega(g, \mathbf{x} ; \delta))^{q} \mathrm{~d} \mathbf{x}\right)^{1 / q} \\
1 \leq q \leq \infty, \delta \in\left[0, d_{\max }\right] \text { and }
\end{gathered}
$$

$\omega(g, \mathbf{x} ; \delta)=\sup \left\{\left|\Delta_{h} g(\mathrm{t})\right|: \mathrm{t}, \mathrm{t}+h \in[\mathbf{x}-\delta / 2, x+\delta / 2] \cap \Omega\right\}$,

where $\Delta_{h}$ is the difference (forward) of the function $g$ in the point $t$ from first order, which in this case plays a role similar to the derivative but in a discrete sense.

Similar to the one obtained by Dupach, a higher order variance estimate can also be derived for a class of functions whose higher order derivatives are constrained by adding additional conditions for the choice of random points in the subdomains. Rayna Georgieva proves the following theorem [5]:

Theorem 3: Let we have domain $\Omega \in \mathbf{R}^{s}$ and the division of the domain $\Omega=\sum_{j=1}^{M} \Omega_{j}$, where every subdomain $\Omega_{j}$ is centrally symmetric with center $p_{j}$. Let $\xi^{(j)}$ is a random $s$ dimensional point, uniformly distributed in $\Omega_{j}, \xi^{(j)^{\prime}}$ is symmetric (about the center $p_{j}$ ) with $\xi^{(j)}$, i.e. $\xi^{(j)}+\xi^{(j)^{\prime}}=2 p_{j}$. If the first and second partial derivatives of a function $g(\mathbf{x})$ are continuous in $\Omega$ and for all $k, l=1, \ldots, s$ is fulfilled that

$$
\frac{\partial^{2} g}{\partial x_{k} \partial x_{l}} \leq L \quad d_{j}=\sup _{x_{1}, x_{2} \in \Omega_{j}}\left|x_{1}-x_{2}\right| \leq \frac{c_{1}}{N^{1 / s}},
$$

then $\mathbf{D} \bar{\theta}_{N}^{* *}=c_{2} L^{2} N^{-1-4 / s}$, where $\bar{\theta}_{N}^{* *}=\sum_{j=1}^{N} \frac{V_{j}}{2}\left[g\left(\xi^{(j)}\right)+\right.$ $\left.g\left(\xi^{(j)^{\prime}}\right)\right], V_{j}$ is the volume of the subdomain $\Omega_{j} \quad c=$ $\left(0.5 s^{2} c_{1}^{s+2}\right)^{2}$

There are various Monte Carlo approaches for numerical integration whose convergence order is $\mathcal{O}\left(N^{-\frac{1}{2}-\frac{k}{s}}\right)$. For $k=1$ and $k=2$ these methods can be constructed relatively easily [2], following Dupach's ideas for $k=1$, described above (see the Theorem 1). But the situation changes when $k \geq 3$. Using the method of controlling the variance on interpolation polynomials, Atanasov and Dimov [1] formulate conditions for constructing a method with optimal order of convergence for $s$-dimensional functions from the class $W^{k}$. The optimal method requires $m * n^{s}$ points and is briefly described below [1].

For $n, s, k \geq 1$ we define integration formula of type Monte Carlo, depends on an integer parameter $m \geq 1$ and $\left(\begin{array}{c}s+k-1 \\ s\end{array}\right)$ points in $[0,1]^{s}$ by the following way. Points $x^{(r)}$ are exactly a number of $\left({ }^{s+k-1}\right)$ and they must fulfil the condition, that if for one polynom $P(x)$, so that for the degree of the polynom $\operatorname{deg} P \leq k$ is fulfilled $P\left(x^{(r)}\right)=0$, then $P \equiv 0$. If $N=n^{s}$ for $n \geq 1$ we divide $s$ dimensional hypercube $[0,1]^{s}$ into $n^{s}$ endless undercubes $K_{j}$, i.e. $[0,1]^{s}=c_{i=1}^{n^{s}} K_{j}$ and $K_{j}=\prod_{i=1}^{s}\left[a_{i}^{j}, b_{i}^{j}\right)$, where $b_{i}^{j}-a_{i}^{j}=\frac{1}{n}$ for every $i=1, \ldots, s$. For every cube $K_{j}$ we calculate the coordinates of $\left(\begin{array}{c}s+k-1 \\ s\end{array}\right)$ points $y^{(r)}$, defined by $y_{i}^{(r)}=a_{i}^{r}+\frac{1}{n} x_{i}(r)$. We assume that we choose $m$ random points $\xi_{i}(j, s)=\left(\xi_{1}(j, p), \ldots, \xi_{s}(j, p)\right)$ from every cube $K_{j}$, such that all the points $\xi_{i}(j, p)$ are independent uniformly distributed random points, we calculate all $f\left(y^{(r)}\right)$ and $f\left(\xi_{i}(j, p)\right)$, and we calculate the Lagrange polynom of the function $f$ in the point $z$ using information about the functional values in the points $y^{(r)}$. We denote the polynom by $L_{k}(f, z)$. For every polynom $P$ of max degree $k-1$ we have that $L_{k}(f, z) \equiv z$. Now we approximate

$\int_{K_{j}} f(x) d x \approx \frac{1}{m n^{s}} \sum_{s=1}^{m}\left[(\xi(j, p))-L_{k}(f, \xi(j, p))\right]+\int_{K_{j}} L_{k}(f, x) d x$.

After that we sum for every $j=1, \ldots, N$ and obtain:

$I(f) \approx \frac{1}{m n^{s}} \sum_{j=1}^{N} \sum_{s=1}^{m}\left[(\xi(j, p))-L_{k}(f, \xi(j, p))\right]+\sum_{j=1}^{N} \int_{K_{j}} L_{k}(f, x) d x$.

$\int_{K_{j}} f(x) d x \approx \frac{1}{m n^{s}} \sum_{s=1}^{m}\left[(\xi(j, p))-L_{k}(f, \xi(j, p))\right]+\int_{K_{j}} L_{k}(f, x) d x$.

After that we sum for every $j=1, \ldots, N$ and obtain:

$I(f) \approx \frac{1}{m n^{s}} \sum_{j=1}^{N} \sum_{s=1}^{m}\left[(\xi(j, p))-L_{k}(f, \xi(j, p))\right]+\sum_{j=1}^{N} \int_{K_{j}} L_{k}(f, x) d x$.

\section{NUMERICAL EXAMPLES}

We will test the optimal method on multidimensional integrals of smooth functions of different dimensions. We use the following notations: $\mathrm{A}==$ Adaptive stochastic approach, $\mathrm{L}=$ Latin Hypercube sampling, $\mathrm{S}=$ Sobol quasi-random sequence, $\mathrm{F}=$ Fibonacci lattice rule, $\mathrm{O}=$ optimal approach. For more information about the description of the different stochastic approaches, see [2], [6]. We must emphasise 
Table I

RELATIVE ERROR FOR THE 3 DIMENSIONAL INTEGRAL DIMENSIONAL INTEGRAL

\begin{tabular}{|c|c|c|c|c|c|c|c|c|c|c|}
\hline \hline $\mathrm{N}$ & $\mathrm{A}$ & $\mathrm{t}$ & $\mathrm{F}$ & $\mathrm{t}$ & $\mathrm{S}$ & $\mathrm{t}$ & $\mathrm{L}$ & $\mathrm{t}$ & $\mathrm{O}$ & $\mathrm{t}$ \\
\hline $10^{3}$ & $4.82 \mathrm{e}-3$ & 0.17 & $1.21 \mathrm{e}-3$ & 0.006 & $4.87 \mathrm{e}-4$ & 0.47 & $6.14 \mathrm{e}-3$ & 0.004 & $3.12 \mathrm{e}-5$ & 0.81 \\
\hline $10^{4}$ & $1.07 \mathrm{e}-3$ & 1.44 & $5.04 \mathrm{e}-4$ & 0.07 & $1.56 \mathrm{e}-4$ & 1.88 & $6.56 \mathrm{e}-4$ & 0.06 & $2.05 \mathrm{e}-6$ & 4.13 \\
\hline $10^{5}$ & $1.52 \mathrm{e}-4$ & 10.9 & $5.34 \mathrm{e}-6$ & 0.66 & $2.51 \mathrm{e}-5$ & 15.6 & $1.34 \mathrm{e}-4$ & 0.51 & $4.58 \mathrm{e}-7$ & 31.62 \\
\hline $10^{6}$ & $5.11 \mathrm{e}-5$ & 131 & $7.85 \mathrm{e}-7$ & 7.02 & $7.43 \mathrm{e}-6$ & 105.80 & $6.84 \mathrm{e}-5$ & 5.22 & $6.72 \mathrm{e}-8$ & 155 \\
\hline $10^{7}$ & $2.34 \mathrm{e}-5$ & 1094 & $8.89 \mathrm{e}-8$ & 79.7 & $1.58 \mathrm{e}-6$ & 934 & $1.73 \mathrm{e}-5$ & 17 & $5.34 \mathrm{e}-9$ & 1053 \\
\hline
\end{tabular}

Table II

RELATIVE ERROR FOR THE 3 DIMENSIONAL INTEGRAL

\begin{tabular}{|c|c|c|c|c|c|c|}
\hline \hline time $(\mathrm{s})$ & $\mathrm{C}$ & $\mathrm{A}$ & $\mathrm{F}$ & S & L & O \\
\hline 1 & $1.05 \mathrm{e}-3$ & $7.96 \mathrm{e}-3$ & $2.34 \mathrm{e}-6$ & $2.93 \mathrm{e}-4$ & $5.11 \mathrm{e}-4$ & $1.21 \mathrm{e}-5$ \\
\hline 5 & $6.84 \mathrm{e}-4$ & $8.14 \mathrm{e}-4$ & $8.47 \mathrm{e}-7$ & $8.01 \mathrm{e}-5$ & $7.32 \mathrm{e}-5$ & $1.12 \mathrm{e}-6$ \\
\hline 10 & $4.79 \mathrm{e}-4$ & $1.82 \mathrm{e}-4$ & $4.89 \mathrm{e}-7$ & $4.71 \mathrm{e}-5$ & $4.32 \mathrm{e}-5$ & $7.21 \mathrm{e}-7$ \\
\hline 100 & $1.57 \mathrm{e}-4$ & $7.04 \mathrm{e}-5$ & $6.53 \mathrm{e}-9$ & $7.68 \mathrm{e}-6$ & $5.32 \mathrm{e}-6$ & $8.61 \mathrm{e}-8$ \\
\hline \hline
\end{tabular}

on the fact this is the first time a comprehensive experimental study with this optimal approach and other stochastic methods under consideration has been done.

Example 1. s=3.

$$
\int_{[0,1]^{3}} \exp \left(x_{1} x_{2} x_{3}\right) \approx 1.14649907 .
$$

Example 2. $\mathrm{s}=4$.

$$
\int_{[0,1]^{4}} x_{1} x_{2}^{2} e^{x_{1} x_{2}} \sin \left(x_{3}\right) \cos \left(x_{4}\right) \approx 0.1089748630 .
$$

Example 3. $\mathrm{s}=5$.

$$
\int_{[0,1]^{5}} \exp \left(-100 x_{1} x_{2} x_{3}\right)\left(\sin \left(x_{4}\right)+\cos \left(x_{5}\right)\right) \approx 0.1854297367
$$

Example 4. $\mathrm{s}=7$.

$$
\int_{[0,1]^{7}} e^{1-\sum_{i=1}^{3} \sin \left(\frac{\pi}{2} \cdot x_{i}\right)} \cdot \arcsin \left(\sin (1)+\frac{\sum_{j=1}^{7} x_{j}}{200}\right) \approx 0.75151101 .
$$

Example 5. $\mathrm{s}=15$.

$$
\int_{[0,1]^{15}}\left(\sum_{i=1}^{10} x_{i}^{2}\right)\left(x_{11}-x_{12}^{2}-x_{13}^{3}-x_{14}^{4}-x_{15}^{5}\right)^{2} \approx 1.96440666 .
$$

Example 6. $s=25$.

$$
\int_{[0,1]^{2} 5} \frac{4 x_{1} x_{3}^{2} e^{2 x_{1} x_{3}}}{\left(1+x_{2}+x_{4}\right)^{2}} e^{x_{5}+\cdots+x_{20}} x_{21} \ldots x_{25} \approx 108.808 .
$$

Example 7. $\mathrm{s}=30$.

$$
\int_{[0,1]^{3} 0} \frac{4 x_{1} x_{3}^{2} e^{2 x_{1} x_{3}}}{\left(1+x_{2}+x_{4}\right)^{2}} e^{x_{5}+\cdots+x_{20}} x_{21} \ldots x_{30} \approx 3.244540 .
$$

For smooth integrands without computational peculiarities Fibonacci lattice algorithm gives better results for lower dimensions,
Table III

RELATIVE ERROR FOR THE 4 DIMENSIONAL INTEGRAL

\begin{tabular}{|c|c|c|c|c|c|c|c|c|c|c|}
\hline $\mathrm{N}$ & $\mathrm{A}$ & $\mathrm{t}$ & $\mathrm{F}$ & $\mathrm{t}$ & $\mathrm{S}$ & $\mathrm{t}$ & $\mathrm{L}$ & $\mathrm{t}$ & $\mathrm{O}$ & $\mathrm{t}$ \\
\hline $10^{4}$ & $3.11 \mathrm{e}-4$ & 1.97 & $2.61 \mathrm{e}-5$ & 0.07 & $2.61 \mathrm{e}-5$ & 2.14 & $5.29 \mathrm{e}-4$ & 0.07 & $1.52 \mathrm{e}-5$ & 4.81 \\
\hline $10^{5}$ & $2.44 \mathrm{e}-5$ & 20.1 & $5.62 \mathrm{e}-6$ & 0.99 & $5.93 \mathrm{e}-6$ & 17.6 & $3.56 \mathrm{e}-4$ & 0.60 & $7.96 \mathrm{e}-6$ & 45.1 \\
\hline $10^{6}$ & $1.13 \mathrm{e}-5$ & 210 & $5.86 \mathrm{e}-7$ & 5.22 & $1.51 \mathrm{e}-6$ & 193 & $4.36 \mathrm{e}-5$ & 4.97 & $2.31 \mathrm{e}-7$ & 352.6 \\
\hline $10^{7}$ & $8.11 \mathrm{e}-6$ & 2035 & $8.38 \mathrm{e}-9$ & 58 & $8.30 \mathrm{e}-7$ & 1121 & $8.12 \mathrm{e}-6$ & 47.1 & $8.16 \mathrm{e}-9$ & 2651 \\
\hline
\end{tabular}

Table IV

RELATIVE ERROR FOR THE 4 DIMENSIONAL INTEGRAL

\begin{tabular}{|c|c|c|c|c|c|c|}
\hline \hline time(s) & C & A & F & S & L & O \\
\hline 0.1 & $3.48 \mathrm{e}-3$ & $3.59 \mathrm{e}-4$ & $1.44 \mathrm{e}-5$ & $4.07 \mathrm{e}-4$ & $4.18 \mathrm{e}-4$ & $4.22 \mathrm{e}-5$ \\
\hline 1 & $1.48 \mathrm{e}-3$ & $2.85 \mathrm{e}-4$ & $5.62 \mathrm{e}-6$ & $3.54 \mathrm{e}-5$ & $3.32 \mathrm{e}-4$ & $2.31 \mathrm{e}-5$ \\
\hline 5 & $6.62 \mathrm{e}-4$ & $9.18 \mathrm{e}-5$ & $5.38 \mathrm{e}-7$ & $5.26 \mathrm{e}-5$ & $4.23 \mathrm{e}-5$ & $1.12 \mathrm{e}-5$ \\
\hline 10 & $2.52 \mathrm{e}-4$ & $1.36 \mathrm{e}-5$ & $3.77 \mathrm{e}-7$ & $6.50 \mathrm{e}-6$ & $3.48 \mathrm{e}-5$ & $7.53 \mathrm{e}-6$ \\
\hline 20 & $1.58 \mathrm{e}-4$ & $2.08 \mathrm{e}-5$ & $2.67 \mathrm{e}-8$ & $4.55 \mathrm{e}-6$ & $2.16 \mathrm{e}-5$ & $6.54 \mathrm{e}-7$ \\
\hline
\end{tabular}

but it requires more random points when the dimensionality increases. The MC algorithm based on Latin hypercube sampling has higher accuracy for this case study with increasing the dimensionality of the integral. The adaptive MC algorithm is slower, but it requires smaller number of random points to achieve better accuracy even for higher dimensions. The efficiency of the optimal $\mathrm{C}$ algorithm is clearly seen for lower dimensions.

As the dimension increases, the computational time of the optimal method decrease due to the increased computational time of the Lagrangian interpolation polynomial. But for very high dimensions 25 and 30 the relative error for a fixed computational time is better than this achieved by the other stochastic methods. From the Tables it is obvious that for small dimensions the FIBO and Optimal method achieves the best accuracy for a fixed preliminary given time - see Tables II,IV,VI. For dimensions up to 10, the FIBO and Optimal methods achieve the smallest relative error for a given number of samples - see Tables I,III,V. The Optimal method is with 1 order better than the FIBO method for a fixxed number of samples, but the advantage of FIBO method is the significantly lower computational time. It is seen that for dimensions 10 and 15, the Optimal method gives relative errors for a fixed number of samples with at least 1

Table V

RELATIVE ERROR FOR THE 5 DIMENSIONAL INTEGRAL

\begin{tabular}{|c|c|c|c|c|c|c|c|c|c|c|}
\hline \hline $\mathrm{N}$ & $\mathrm{A}$ & $\mathrm{t}$ & $\mathrm{F}$ & $\mathrm{t}$ & $\mathrm{S}$ & $\mathrm{t}$ & $\mathrm{L}$ & $\mathrm{t}$ & $\mathrm{O}$ & $\mathrm{t}$ \\
\hline $10^{3}$ & $2.15 \mathrm{e}-3$ & 0.27 & $1.75 \mathrm{e}-4$ & 0.007 & $5.29 \mathrm{e}-4$ & 0.03 & $9.38 \mathrm{e}-3$ & 0.007 & $2.75 \mathrm{e}-5$ & 2.1 \\
\hline $10^{4}$ & $2.01 \mathrm{e}-3$ & 2.43 & $1.28 \mathrm{e}-5$ & 0.06 & $1.43 \mathrm{e}-4$ & 0.3 & $3.44 \mathrm{e}-3$ & 0.07 & $7.22 \mathrm{e}-6$ & 2.3 \\
\hline $10^{5}$ & $6.91 \mathrm{e}-4$ & 22.2 & $9.50 \mathrm{e}-6$ & 0.61 & $2.36 \mathrm{e}-5$ & 2.77 & $2.01 \mathrm{e}-3$ & 0.69 & $2.36 \mathrm{e}-6$ & 6.2 \\
\hline $10^{6}$ & $2.92 \mathrm{e}-4$ & 219.5 & $5.47 \mathrm{e}-7$ & 5.98 & $6.07 \mathrm{e}-6$ & 24.2 & $1.80 \mathrm{e}-4$ & 6.17 & $5.46 \mathrm{e}-7$ & 20.0 \\
\hline $10^{7}$ & $8.21 \mathrm{e}-5$ & 2043 & $8.71 \mathrm{e}-9$ & 58.4 & $2.30 \mathrm{e}-6$ & 245 & $2.46 \mathrm{e}-5$ & 60.5 & $7.01 \mathrm{e}-8$ & 105.1 \\
\hline
\end{tabular}

Table VI

RELATIVE ERROR FOR THE 5 DIMENSIONAL INTEGRAL

\begin{tabular}{|c|c|c|c|c|c|}
\hline \hline time(s) & A & F & S & L & O \\
\hline 0.1 & $3.16 \mathrm{e}-3$ & $3.48 \mathrm{e}-3$ & $1.09 \mathrm{e}-5$ & $1.34 \mathrm{e}-4$ & $3.21 \mathrm{e}-3$ \\
\hline 1 & $1.08 \mathrm{e}-3$ & $2.08 \mathrm{e}-3$ & $5.58 \mathrm{e}-6$ & $7.21 \mathrm{e}-5$ & $8.54 \mathrm{e}-4$ \\
\hline 5 & $8.79 \mathrm{e}-4$ & $8.20 \mathrm{e}-4$ & $8.71 \mathrm{e}-7$ & $1.54 \mathrm{e}-5$ & $3.25 \mathrm{e}-4$ \\
\hline 10 & $5.85 \mathrm{e}-4$ & $7.51 \mathrm{e}-4$ & $4.15 \mathrm{e}-7$ & $9.32 \mathrm{e}-6$ & $8.65 \mathrm{e}-5$ \\
\hline 20 & $3.99 \mathrm{e}-4$ & $6.95 \mathrm{e}-4$ & $8.37 \mathrm{e}-8$ & $7.39 \mathrm{e}-6$ & $5.02 \mathrm{e}-5$ \\
\hline
\end{tabular}


Table VII

RELATIVE ERROR FOR THE 7 DIMENSIONAL INTEGRAL

\begin{tabular}{|c|c|c|c|c|c|c|c|c|c|c|}
\hline \hline $\mathrm{N}$ & $\mathrm{A}$ & $\mathrm{t}$ & $\mathrm{F}$ & $\mathrm{t}$ & $\mathrm{S}$ & $\mathrm{t}$ & $\mathrm{L}$ & $\mathrm{t}$ & $\mathrm{O}$ & $\mathrm{t}$ \\
\hline $10^{4}$ & $1.07 \mathrm{e}-3$ & 2.07 & $2.19 \mathrm{e}-3$ & 0.11 & $2.27 \mathrm{e}-4$ & 0.76 & $1.79 \mathrm{e}-3$ & 0.13 & $2.13 \mathrm{e}-4$ & 10.2 \\
\hline $10^{5}$ & $7.51 \mathrm{e}-4$ & 19.3 & $6.19 \mathrm{e}-4$ & 0.99 & $1.22 \mathrm{e}-4$ & 7.45 & $2.53 \mathrm{e}-4$ & 1.15 & $4.41 \mathrm{e}-5$ & 40.2 \\
\hline $10^{6}$ & $6.30 \mathrm{e}-5$ & 194 & $1.99 \mathrm{e}-5$ & 9.81 & $4.71 \mathrm{e}-5$ & 72.3 & $8.27 \mathrm{e}-5$ & 10.32 & $1.27 \mathrm{e}-6$ & 167.1 \\
\hline $10^{7}$ & $2.34 \mathrm{e}-5$ & 1861 & $4.89 \mathrm{e}-7$ & 94.2 & $9.45 \mathrm{e}-6$ & 697 & $1.69 \mathrm{e}-5$ & 101.2 & $1.45 \mathrm{e}-7$ & 595.1 \\
\hline \hline
\end{tabular}

Table VIII

RELATIVE ERROR FOR THE 7 DIMENSIONAL INTEGRAL

\begin{tabular}{|c|c|c|c|c|c|}
\hline \hline time(s) & A & F & S & L & O \\
\hline 0.1 & $2.38 \mathrm{e}-2$ & $3.11 \mathrm{e}-2$ & $2.38 \mathrm{e}-3$ & $1.85 \mathrm{e}-3$ & $2.37 \mathrm{e}-3$ \\
\hline 1 & $8.87 \mathrm{e}-3$ & $2.88 \mathrm{e}-3$ & $6.19 \mathrm{e}-4$ & $1.85 \mathrm{e}-4$ & $3.37 \mathrm{e}-4$ \\
\hline 5 & $5.16 \mathrm{e}-3$ & $3.76 \mathrm{e}-3$ & $8.81 \mathrm{e}-5$ & $9.79 \mathrm{e}-5$ & $1.38 \mathrm{e}-4$ \\
\hline 10 & $1.28 \mathrm{e}-3$ & $6.71 \mathrm{e}-4$ & $1.88 \mathrm{e}-5$ & $8.36 \mathrm{e}-5$ & $8.78 \mathrm{e}-5$ \\
\hline 20 & $2.03 \mathrm{e}-3$ & $4.28 \mathrm{e}-4$ & $3.87 \mathrm{e}-6$ & $5.46 \mathrm{e}-5$ & $6.87 \mathrm{e}-5$ \\
\hline
\end{tabular}

Table IX

RELATIVE ERROR FOR THE 15 DIMENSIONAL INTEGRAL

\begin{tabular}{|c|c|c|c|c|c|c|c|c|c|c|}
\hline \hline $\mathrm{N}$ & $\mathrm{A}$ & $\mathrm{t}$ & $\mathrm{F}$ & $\mathrm{t}$ & $\mathrm{S}$ & $\mathrm{t}$ & $\mathrm{L}$ & $\mathrm{t}$ & $\mathrm{O}$ & $\mathrm{t}$ \\
\hline $10^{3}$ & $3.16 \mathrm{e}-3$ & 9.24 & $5.34 \mathrm{e}-2$ & 0.08 & $2.04 \mathrm{e}-3$ & 0.98 & $1.06 \mathrm{e}-2$ & 0.12 & $7.54 \mathrm{e}-3$ & 27.4 \\
\hline $10^{4}$ & $1.49 \mathrm{e}-3$ & 88 & $1.22 \mathrm{e}-3$ & 0.93 & $2.89 \mathrm{e}-4$ & 9.3 & $7.33 \mathrm{e}-3$ & 1.07 & $6.51 \mathrm{e}-4$ & 81.5 \\
\hline $10^{5}$ & $5.76 \mathrm{e}-4$ & 847 & $3.08 \mathrm{e}-4$ & 9.65 & $1.13 \mathrm{e}-4$ & 93.8 & $1.54 \mathrm{e}-3$ & 10.11 & $7.29 \mathrm{e}-5$ & 242.1 \\
\hline $10^{6}$ & $1.29 \mathrm{e}-4$ & 8235 & $1.37 \mathrm{e}-5$ & 96.9 & $1.93 \mathrm{e}-5$ & 935 & $1.14 \mathrm{e}-4$ & 99.6 & $8.29 \mathrm{e}-6$ & 720.2 \\
\hline \hline
\end{tabular}

order better than FIBO and Sobol, see Tables VII and IX, with FIBO

Table X

RELATIVE ERROR FOR THE 15 DIMENSIONAL INTEGRAL

\begin{tabular}{|c|c|c|c|c|c|}
\hline \hline time(s) & $\mathrm{A}$ & $\mathrm{F}$ & $\mathrm{S}$ & $\mathrm{L}$ & $\mathrm{O}$ \\
\hline 1 & $9.96 \mathrm{e}-1$ & $6.30 \mathrm{e}-3$ & $1.10 \mathrm{e}-3$ & $3.64 \mathrm{e}-3$ & $3.51 \mathrm{e}-2$ \\
\hline 5 & $7.72 \mathrm{e}-2$ & $1.68 \mathrm{e}-3$ & $2.45 \mathrm{e}-4$ & $7.32 \mathrm{e}-4$ & $1.23 \mathrm{e}-2$ \\
\hline 10 & $1.33 \mathrm{e}-2$ & $5.89 \mathrm{e}-3$ & $9.48 \mathrm{e}-5$ & $1.94 \mathrm{e}-4$ & $9.63 \mathrm{e}-3$ \\
\hline 20 & $3.03 \mathrm{e}-2$ & $1.66 \mathrm{e}-3$ & $9.87 \mathrm{e}-6$ & $4.05 \mathrm{e}-5$ & $7.51 \mathrm{e}-3$ \\
\hline 100 & $8.11 \mathrm{e}-3$ & $5.04 \mathrm{e}-4$ & $8.17 \mathrm{e}-7$ & $4.03 \mathrm{e}-6$ & $9.51 \mathrm{e}-5$ \\
\hline \hline
\end{tabular}

Table XI

RELATIVE ERROR FOR THE 25 DIMENSIONAL INTEGRAL

\begin{tabular}{|c|c|c|c|c|c|c|}
\hline \hline $\mathrm{N}$ & $\mathrm{O}$ & $\mathrm{t}, \mathrm{s}$ & $\mathrm{S}$ & $\mathrm{t}, \mathrm{s}$ & $\mathrm{L}$ & $\mathrm{t}, \mathrm{s}$ \\
\hline $10^{3}$ & $3.77 \mathrm{e}-3$ & 2.03 & $1.47 \mathrm{e}-1$ & 0.4 & $7.54 \mathrm{e}-1$ & 0.02 \\
\hline $10^{4}$ & $3.18 \mathrm{e}-3$ & 19.52 & $5.68 \mathrm{e}-2$ & 5.64 & $5.39 \mathrm{e}-2$ & 0.15 \\
\hline $10^{5}$ & $5.33 \mathrm{e}-5$ & 181 & $7.21 \mathrm{e}-3$ & 33.4 & $2.11 \mathrm{e}-2$ & 1.07 \\
\hline $10^{6} 3$ & $3.11 \mathrm{e}-5$ & 1234 & $2.89 \mathrm{e}-3$ & 161 & $1.71 \mathrm{e}-4$ & 8.21 \\
\hline \hline
\end{tabular}

Table XII

RELATIVE ERROR FOR THE 25 DIMENSIONAL INTEGRAL

\begin{tabular}{|c|c|c|c|}
\hline \hline time,sec & $\mathrm{O}$ & $\mathrm{S}$ & $\mathrm{L}$ \\
\hline 1 & $7.24 \mathrm{e}-2$ & $9.51 \mathrm{e}-2$ & $2.11 \mathrm{e}-2$ \\
\hline 5 & $8.16 \mathrm{e}-3$ & $5.76 \mathrm{e}-2$ & $1.61 \mathrm{e}-2$ \\
\hline 10 & $5.18 \mathrm{e}-3$ & $2.71 \mathrm{e}-2$ & $9.58 \mathrm{e}-3$ \\
\hline 20 & $3.13 \mathrm{e}-3$ & $8.28 \mathrm{e}-3$ & $7.87 \mathrm{e}-3$ \\
\hline \hline
\end{tabular}

Table XIII

RELATIVE ERROR FOR 30 DIMENSIONAL INTEGRAL

\begin{tabular}{|c|c|c|c|c|c|c|}
\hline \hline $\mathrm{N}$ & $\mathrm{O}$ & $\mathrm{t}, \mathrm{s}$ & $\mathrm{S}$ & $\mathrm{t}, \mathrm{s}$ & $\mathrm{L}$ & $\mathrm{t}, \mathrm{s}$ \\
\hline $10^{3}$ & $2.01 \mathrm{e}-2$ & 5.4 & $1.18 \mathrm{e}-1$ & 0.42 & $8.81 \mathrm{e}-1$ & 0.02 \\
\hline $10^{4}$ & $6.53 \mathrm{e}-3$ & 14.5 & $8.40 \mathrm{e}-2$ & 4.5 & $6.19 \mathrm{e}-2$ & 0.14 \\
\hline $10^{5}$ & $1.35 \mathrm{e}-3$ & 145 & $1.18 \mathrm{e}-2$ & 30.2 & $2.78 \mathrm{e}-2$ & 1.16 \\
\hline $10^{6}$ & $2.11 \mathrm{e}-4$ & 1290 & $9.20 \mathrm{e}-3$ & 168 & $9.86 \mathrm{e}-3$ & 8.61 \\
\hline \hline
\end{tabular}

Table XIV

RELATIVE ERROR FOR 30 DIMENSIONAL INTEGRAL

\begin{tabular}{|c|c|c|c|}
\hline \hline time,sec & $\mathrm{O}$ & $\mathrm{S}$ & $\mathrm{L}$ \\
\hline 1 & $4.38 \mathrm{e}-1$ & $1.01 \mathrm{e}-1$ & $2.38 \mathrm{e}-2$ \\
\hline 5 & $1.16 \mathrm{e}-2$ & $7.76 \mathrm{e}-2$ & $1.81 \mathrm{e}-2$ \\
\hline 10 & $8.11 \mathrm{e}-3$ & $5.71 \mathrm{e}-2$ & $9.48 \mathrm{e}-3$ \\
\hline 20 & $4.63 \mathrm{e}-3$ & $1.28 \mathrm{e}-2$ & $7.87 \mathrm{e}-3$ \\
\hline
\end{tabular}

achieving better accuracy in much less time - see Tables VIII and X. The adaptive algorithm is suitable for higher dimensions because of the small number of samples to achieve the preliminary given relative error. The Adaptive algorithm performs better than FIBO and Sobol - see Tables XI and XIII. Analyzes show that for high 25 and 30 samples, the Optimal MC approach outperforms LHS and Sobol's quasi-Monte Carlo algorithm - see Tables XII and XIV, where the LHS method produce better results than the Sobol QMC approach. Here we do not test Adaptive approach, because of the significant amount of time and FIBO method which gives unreliable relative errors for the high dimensional case.

\section{CONCLUSIONS}

The efficiency of the optimal Monte Carlo algorithm for the calculation of multidimensional integrals has been presented. The optimal approach appears to be an efficient stochastic solution to solve this kind of problem, because we demonstrate here its reliability for calculating integrals with smooth integrands in a relatively small subregion of the original integration domain regardless of the dimension.

\section{REFERENCES}

[1] E. Atanassov and I.T. Dimov, A new optimal monte carlo method for calculating integrals of smooth functions, Journal of Monte Carlo Methods and Applications 5 (1999), no. 2, 149-167, https://doi.org/10.1515/mcma.1999.5.2.149.

[2] Dimov I. (2008) Monte Carlo Methods for Applied Scientists, New Jersey, London, Singapore, World Scientific, 291 p., ISBN-10 981-022329-3.

[3] I.T. Dimov and O. Tonev, Monte carlo algorithms: performance analysis for some computer architectures, J. of Computational and Applied Mathematics 48 (1993), 253-277, https://doi.org/10.1016/03770427(93)90024-6.

[4] V. Dupach, Stochasticke pocetni metody, Cas. pro pest. mat. 81 (1956), no. $1,55-68$.

[5] Georgieva, R.: PhD Thesis: Computational complexity of Monte Carlo algorithms for multidimensional integrals and integral equations, Sofia, 2003

[6] I.M. Sobol, (1989) Quasi-Monte Carlo methods, in: Sendov, Bl., Dimov, I.T. (eds.) International Youth Workshop on Monte Carlo Methods and Parallel Algorithms, World Scientific, Singapore: 75-81. 